\title{
The importance of inner-shell electronic structure for enhancing the EUV absorption of photoresist materials
}

Kristina D. Closser, D. Frank Ogletree, Patrick Naulleau, and David Prendergast

Lawrence Berkeley National Laboratory, 1 Cyclotron Road, Berkeley, California 94720

\begin{abstract}
In order to increase computation power and efficiency, the semiconductor industry continually strives to reduce the size of features written using lithographic techniques. The planned switch to a shorter wavelength extreme ultraviolet (EUV) source presents a challenge for the associated photoresists, which in their current manifestation show much poorer photoabsorption cross sections for the same dose. Here we consider the critical role that an inner-shell electronic structure might play in enhancing photoabsorption cross sections, which one can control by the choice of substituent elements in the photoresist. In order to increase the EUV sensitivity of current photoresists, it is critical to consider the inner-shell atomic structure of the elements that compose the materials. We validate this hypothesis using a series of halogenated organic molecules, which all have similar valence structures, but differ in the character of their semi-core and deep valence levels. Using various implementations of time-dependent density functional theory, the absorption cross sections are computed for the model systems of $\mathrm{CH} 3 \mathrm{XCH} 3 \mathrm{X}, \mathrm{X}=\mathrm{H}, \mathrm{OH}, \mathrm{F}, \mathrm{Cl}, \mathrm{Br}, \mathrm{I}, \mathrm{as}$ well as a representative polymer fragment: 2-methyl-phenol and its halogenated analogues. lodine has a particularly high cross section in the EUV range, which is due to delayed absorption by its 4delectrons. The computational results are compared to standard database values and experimental data when available. Generally we find that the states that dominate the EUV oscillator strength are generated by excitations of deep valence or semi-core electrons, which are primarily atomic-like and relatively insensitive to the specific molecular structure.
\end{abstract}

\section{INTRODUCTION}

\section{A. Extreme ultraviolet (EUV) lithography}

Photolithography is a cost-efficient method for manufacturing integrated circuits. In this process, a surface coated with a photoresist and illuminated through a projection mask is exposed

to radiation. The photoresist undergoes chemical changes where exposed to light, becoming more soluble by fragmenting or less soluble through cross-linking or polymerization, for positive or negative tone resists, respectively. Current industrial processing uses deep ultraviolet (DUV) radiation at $193 \mathrm{~nm}(6.4 \mathrm{eV})$, but in order to form smaller features without resorting to time consuming and costly multiple patterning techniques, shorter wavelengths are required. Smaller features have faster responses and form denser integrated circuits, leading to more energy efficient and cheaper devices. The photolithography industry is currently focusing on the next generation of light sources which produce EUV radiation at $13.5 \mathrm{~nm}(92 \mathrm{eV})$. EUV is generally considered to encompass the highest energy region of the ultraviolet spectrum between 10 and $100 \mathrm{~nm}$, although for industrial purposes it is exactly $13.5 \mathrm{~nm}$. Such photons have much higher energy than those used in standard UV photolithography, and have the potential to induce very different chemistry, possibly rendering current UV resists obsolete. The interaction of EUV light with photoresist materials is a complex multi-step process; however, after the following brief discussion of the steps involved, this paper will focus only on the initial photon absorption.

The dominant excitation and decay processes in an EUV resist are shown schematically in Fig. 1(a). If the incoming photon is to induce excitations and ultimately chemical changes, it must be absorbed or inelastically scattered. Inelastic scattering can be neglected in a first approximation, as the probability of this process is orders of magnitude lower than that of the resonant absorptionprocess. At $92 \mathrm{eV}$, the photon energy is well above the ionization energy of photoresist materials. For example, traditional organic resists typically have a first ionization energy near $10 \mathrm{eV}$. Electronswith binding energies lower than the photon energy will most likely absorb the photon and be emitted with kinetic energies that are equal to the difference between the photon energies and binding energy. These primary photoelectrons can be produced with up to $80 \mathrm{eV}$ kinetic energy $\left(E_{K}\right)$ in typical organic photoresist materials. For photon energies below the ionization energy of the valence electrons, ionization is not possible, and absorption can only create excitations to bound excited 
states. Thus, $193 \mathrm{~nm}$ (UV) photons, in most cases (assuming intensities consistent with linear absorption, which is true for typical lithographic conditions), cannot directly ionize photoresists, but 13.5 $\mathrm{nm}$ (EUV) photons definitely can and this opens up new reaction channels for the resist materials that are not otherwise accessible.
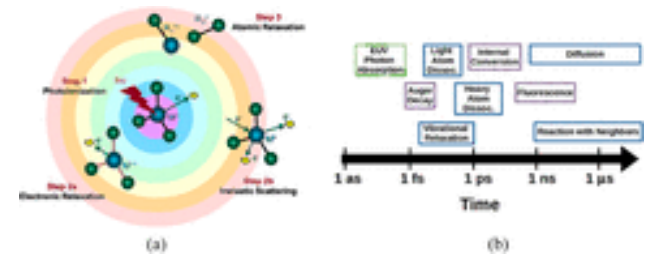

FIG. 1.(a) Cartoon representation of dominant events occurring in EUV absorption and subsequent electronic and nuclear relaxation. (b) Schematic representation of the time scales of processes relevant to EUV excitation and decay. Processes involving electronic relaxation have a purple dashed border and those requiring motion of nuclei have a solid blue border. Auger decay strongly dominates after excitation at EUV energies. Electron motion and relaxation is generally faster than that of the nuclei, allowing the processes to be effectively treated independently.

Optical absorption is proportional to the imaginary part of the polarizability (or susceptibility in condensed phase systems), and this can be expressed as

$$
\alpha(\omega) \propto \sum\left\{\{|\langle i|| \hat{\mu}|| f\rangle| 2 \omega-\omega f i+i \eta+|\langle i|| \hat{\mu}|| f\rangle| 2 \omega+\omega f i+i \eta\}, \alpha(\omega) \propto \sum f\left\{\left\langle i\left|\mu^{\wedge}\right| f\right\rangle|2 \omega-\omega f i+i \eta+|\left\langle i\left|\mu^{\wedge}\right| f\right\rangle \mid 2 \omega+\omega f i+i \eta\right\},\right.
$$

where $\omega \omega$ is the energy of the incoming radiation and $\omega f i=E f-E i \omega f i=E f-E i$ is the energy difference between the final state $f$ and the initial state $i$. Thus, peaks in the absorption spectrum occur when the incoming radiation is resonant with the energy difference between the initial and final states, $\omega=\omega f i \omega=\omega f i$, as well as when the dipolar coupling between the states is large. Absorption cross sections for valence electrons generally peak around $10-20 \mathrm{eV}$, which is a few eV above the ionizationthreshold, and decay rapidly. Additional intensity due to inner-shell excitations begins to appear when the incoming photons have energy that is at least as large as the corresponding energy gap, $\omega \geq \omega \mathrm{fi} \omega \geq \omega \mathrm{fi}$. Generally, absorption peaks appear at energies near the innershell orbital binding energies; however, for some higher values of angular momentum ( $\geq 21 \geq 2$ ), there can be a delayed absorption maximum due to the very weak dipole coupling between the initial and final states at the resonant energy. Thus, if we ignore the fine structure in the resonant but bound near-edge absorption of the deepest energetically accessible orbitals, the most likely fate of these electrons is photoionization by EUV radiation. The resulting holes are most likely to be associated with the removal of deep valence or shallow-core electrons. Note that this is contrary to the behavior for $92 \mathrm{eV}$ electron-induced ionization, where the partial cross sections for the excitation of deeper electrons are significantly smaller than those in the outer valence bands due to screening of the inner electrons (e.g., see Refs. $\underline{12}$ and $\underline{13}$ ). These semi-core excitations are generally highly localized on individual atoms and suggest the potential of designing materials with localized, EUV resonant states. Such states could then be tailored to induce specific local changes in the chemistry and ultimately create resists that better preserve the small scale feature dimensions.

The highly energetic semi-core holes will be filled rapidly $\left(10^{0}-10^{1} \mathrm{fs}\right)$, primarily via Auger decay. In the Auger process, a valence electron fills the semi-core hole and transfers energy to a second electron, which is ejected with characteristic kinetic energies provided the transferred energy is greater than or equal to the corresponding binding energy. Thus, EUV radiation can potentially generate two or more electrons for each photon absorbed, which are then available to do additional work, provided that the corresponding semicore orbitals are present.

Both the primary photoelectron and any secondary electrons generated can interact with the surrounding materials (Step 2b, Fig. 1(a)) potentially causing additional electron-induced ionizationevents (EK>EBEK>EB). Alternatively, the emitted electrons can generate internal excitations (EB $\geq E K>E L U M O-E H O M O E B \geq E K>E L U M O-E H O M O)$ or undergo electron attachment. Note 
that $H O M O$ and $L U M O$ refer to the highest occupied and lowest unoccupied molecular orbitals, respectively, so $E_{L U M O}-E_{H O M O}$ is approximately the energy of the first electronically excited state.

The nuclei will eventually respond to the excitation induced electronic forces and may dissociate or form new bonds resulting in changes to the solubility of the photoresist material (Step 3, Fig. 1(a)). The relevant ionic states are determined by the time scales of the various processes. Electronic relaxation within a band (orbitals near in energy) generally occurs an order of magnitude (or more) faster than the atomic vibrations. The approximate time scales of several of these processes are shown in Fig. $\underline{1}$ (b). Of particular note is that Auger decay $\left(10^{-18}-10^{-15} \mathrm{~s}\right)$ and internal relaxation processes $(\approx 10-12 \approx 10-12 \mathrm{~s})$ are generally much more rapid than fluorescence $\left(10^{-9}-10^{-7} \mathrm{~s}\right), \stackrel{21}{ }$ and also that local chemical changes occur on the time scale of molecular vibrations $\left(10^{-14}-10^{-12} \mathrm{~s}\right)$, while non-local reactions first require diffusion of the generated atoms or ions $(>10-9>10-9 \mathrm{~s})$.

\section{B. Resist materials}

Traditional UV resist materials are organic polymers, comprising $\mathrm{C}, \mathrm{H}$, and $\mathrm{O}$, and sometimes containing elements such as silicon or sulfur. ${ }^{-}$An example of one such resist is shown in Fig. $\underline{2(a)}$. Known as an ESCAP (environmentally stable chemical amplification positive) resist, it was developed by IBM and is a random copolymer formed from 4-acetoxystyrene and t-butyl acrylate. The EUVcross sections of the elements in such organic resists are extremely small and the photoabsorption is only due to excitations of valence electrons. Valence cross sections are orders of magnitude smaller at $92 \mathrm{eV}$ than they are for photons at lower energies used in UV photolithography. Additionally, for an energetically equivalent dose using 92 and $6.4 \mathrm{eV}$ photons, there are roughly 14 times fewer EUV photons; thus, optimizing the absorption from EUV sources, which begin with a much lower flux, is critical to making EUV lithography cost-effective.

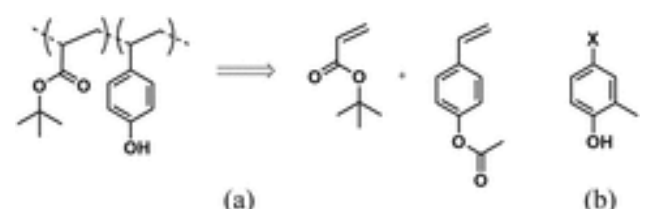

FIG. 2.(a) Example of random block copolymer organic photoresistand monomers used in synthesis. (b) Substituted phenols used in this study $(\mathrm{X}=\mathrm{H}, \mathrm{F}, \mathrm{Cl}, \mathrm{Br}, \mathrm{I})$ as model organic photoresist materials.

Our strategy to increase absorption in the EUV range is to incorporate elements with higher cross sections at $92 \mathrm{eV}$ into the photoresist. These will generally have semi-core levels that are accessible to the EUV radiation. The commonly used atomic data provide an indication of elements to consider. Non-noble gas elements with the highest cross sections are tellurium, iodine, cesium, and astatine; however, there are many others that could also be considered: tin, antimony, and even nickel or copper (Fig. $\underline{3}$ ). Chemically, it is straightforward to incorporate halogens into organic materials, and as iodine has a particularly high cross section, we elected to study the effect of halogenation on traditional UV resists. Both iodine and bromine possess semi-core electrons that may be ionized with EUV, but in fluorine and chlorine, only the valence electrons will contribute to the absorption-cross section. We emphasize that this strategy keeps the chemistry of the photoresist materials similar to the UV resists which are known to pattern, but allows us to explore the effects of incorporating elements with high EUV cross sections. There may also be chemistry useful to lithography involving other high atomic cross section elements, which we are not intending to overlook, but the additional complexity that is introduced by novel materials will need to be considered in future work, and the basic considerations for EUV absorption should be similar. Such novel materials include hafnium or tin oxide nanoparticles as well as various organometallic systems. Additionally, there is not yet an industry consensus on which materials are most promising, so the potential range of chemistry that may be considered is still largely unknown. Thus, any method suggested for use in studying the absorption of EUV materials must 
be applicable to a wide range of condensed phase systems, and computationally efficient so that materials can be screened rapidly for their absorption properties.

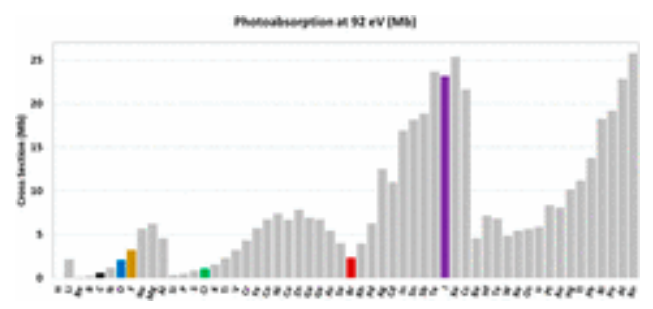

FIG. 3.Photoabsorption cross section at $92 \mathrm{eV}$ for increasing atomic number. Carbon, oxygen, hydrogen, and the halogens discussed in this study are highlighted.

As a model for a functionalized organic photoresist monomer, 2-methyl-4-halogenated phenol was selected (Fig. 2(b)). We begin with a discussion of the available computational models in Section II Aand details of our approach in Section Iㅡ. We first apply the various methodologies to methane and substituted variants, for which experimental data exist, in Section $\underline{\| \mathrm{C}}$. Then the corresponding results for the phenols are presented in Section III A for gas phase molecules and Section 프 B for a condensed phase model. This is followed by a discussion of the validity of using atomic data for predicting the increase in absorption for resist materials due to incorporating high EUV cross section elements (Section III C).

\section{METHODOLOGY}

\section{A. Theoretical background}

Computing absorption spectra at EUV energies for multi-atom systems is a challenging task for theory. This is primarily due to the large number of excited states that must be considered, and that simplifications that may be applied for higher energy X-rays, such as separating the core from the valence orbitals, are not necessarily valid.

The most common approach for computing electronic excitations is using time-dependent density functional theory (TDDFT), which provides a reasonable compromise between accuracy and feasibility for systems consisting of more than a few atoms. Much has been written on TDDFT, including several excellent reviews, which may be consulted for further information.

There are many flavors of TDDFT, which may be divided into two primary classes of linear-response (LR) and real-time (RT) TDDFT. The various approaches used in this paper are briefly outlined in the following paragraphs, and their respective strengths and weaknesses are considered.

1. Linear response

In LR-TDDFT, the linear response of the system to an external field is evaluated using time-dependent perturbation theory, starting from the time-dependent Kohn-Sham (TDKS) equations,

$\mathrm{i} \partial \phi \mathrm{i}(\boldsymbol{r}, \mathrm{t}) \partial \mathrm{t}=\mathrm{H} \mathrm{KS}(\mathrm{t}) \phi \mathrm{i}(\boldsymbol{r}, \mathrm{t}), \mathrm{i} \partial \varphi \mathrm{i}(\mathbf{r}, \mathrm{t}) \partial \mathrm{t}=\mathrm{H}^{\wedge} \mathrm{KS}(\mathrm{t}) \varphi \mathrm{i}(\mathbf{r}, \mathrm{t})$,

where $\hat{\mathrm{H}} \mathrm{KSH} \wedge \mathrm{KS}$ is the Kohn-Sham (KS) Hamiltonian, and фiфi are the KS orbitals. The true one particle density is equivalent to the KS density by the Hohenberg-Kohn theorem and is defined by

$\rho(\boldsymbol{r}, \mathrm{t})=\sum \operatorname{inocc}|\phi i(\boldsymbol{r}, \mathrm{t})| 2 . \rho(\mathbf{r}, \mathrm{t})=\sum \operatorname{inocc}|\varphi \mathrm{i}(\mathbf{r}, \mathrm{t})| 2$.

Using an external perturbation of the form

$V(1)(t)=12 \varepsilon \cdot \hat{\mu}(e i \omega t+e-i \omega t) V^{\wedge}(1)(t)=12 \varepsilon \cdot \mu^{\wedge}(e i \omega t+e-i \omega t)$

and the Brillouin theorem, the first order response of the wavefunction can be written as

$\phi(1) i(t)=\varepsilon 2 \sum a \phi(0) a\{X i a e-i \omega t+Y i a e i \omega t\} \varphi i(1)(t)=\varepsilon 2 \sum a \varphi a(0)\{X i a e-i \omega t+Y i a e i \omega t\}$

so the first order response of the density is 
$\rho(1)=\sum \mathrm{i} \phi *(1) \mathrm{i} \phi(0) \mathrm{i}+\phi *(0) \mathrm{i} \phi(1) \mathrm{i}, \rho(1)=\sum \mathrm{i} \varphi \mathrm{i} *(1) \varphi \mathrm{i}(0)+\varphi \mathrm{i} *(0) \varphi \mathrm{i}(1)$,

$=\varepsilon 2 \sum i a \phi *(0) a \phi(0) i\{X * i a e i \omega t+Y * i a e-i \omega t\}+\phi *(0) i \phi(0) a\{X i a e-i \omega t+Y i a e i \omega t\},=\varepsilon 2 \sum i a \phi a *(0) \phi i(0)\{X i a * e i \omega t+Y i a * e-i \omega t\}+\phi i *(0) \phi a(0)\{X i a e-i c$

and collecting terms allow the LR-TDDFT equations to be written in a simplified matrix form, commonly known as the Casida formalism,

$[A B * B A *][X Y]=\omega[100-1][X Y],[\mathrm{ABB} * \mathbf{A} *][\mathrm{XY}]=\omega[100-1][X Y]$,

where

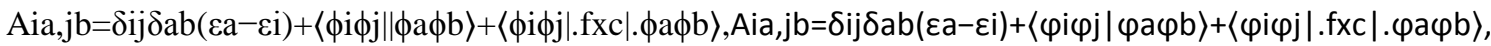

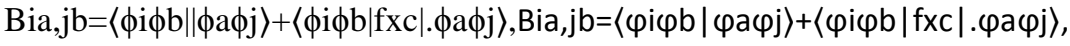

and $f_{x c}$ is the second functional derivative of the exchange-correlation energy (known as the exchangecorrelation kernel). The Tamm-Dancoff approximation (TDA) is equivalent to setting $\mathbf{B}=0$ and simplifies the TDDFT equations to a Hermitian eigenvalue problem,

$\mathbf{A X}=\omega \mathbf{X} \cdot \mathbf{A X}=\omega \mathbf{X}$.

The TDA is very popular within quantum chemistry communities as it reduces the size of the problem and often

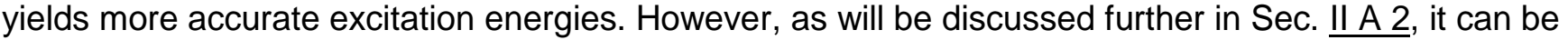
problematic for EUV excited states. To obtain the excitation energies and excited states, one solves for the eigenvalues and eigenvectors of this system. There must be as many solutions as desired states, which rapidly places computational limits on the size of the system that can be treated. Additionally, explicit unoccupied orbitals are required, which places very high demands on the flexibility of the basis set that is needed to represent the high energy virtual orbitals. Formally matrix diagonalization scales as $\mathcal{O}(\mathrm{N} 3) \mathrm{O}(\mathrm{N} 3)$, while the number of possible excitations grows as $\mathcal{O}(\mathrm{N} 2) \mathrm{O}(\mathrm{N} 2)$, where $N$ is a measure of the size of the system.

Although this cost can be reduced by using algorithms such as that by Davidson, such algorithms become less efficient when many states are required. Thus, for EUV regions of the spectrum, where it is often necessary to compute several thousand TDDFT eigenvalues, this method would not be practical for large systems.

The optical absorption spectrum is ultimately given by the optical cross section, which is directly proportional to the excitation frequency times the imaginary part of the frequency dependent polarizability $\alpha(\omega) \alpha(\omega)$,

$\sigma(\omega)=4 \pi \omega c \operatorname{Im}[\alpha(\omega)], \sigma(\omega)=4 \pi \omega c \operatorname{Im}[\alpha(\omega)]$

where $\alpha(\omega) \alpha(\omega)$ can be expressed as a sum over states,

$\alpha(\omega)=3 e 2 \hbar m \sum n f 0 n(E n-E 0) 2, \alpha(\omega)=3 e 2 \hbar m \sum n f 0 n(E n-E 0) 2$,

with $E_{n}$ and $E_{0}$ as the energies of the $n$th excited state and the ground state, respectively, and $f_{0 n}$ is the oscillator strength between state $n$ and the ground state defined as

f0n $=2 m \omega 0 n 3 \hbar \|\left\langle\Psi n \| \mu^{\wedge}|| \Psi 0\right\rangle|| 2 . f 0 n=2 m \omega 0 n 3 \hbar\left|\left\langle\Psi n\left|\mu^{\wedge}\right| \Psi 0\right\rangle\right| 2$.

Within LR-TDDFT, the final state can be expressed as a sum of single particle excitations, so Eq. (14)can be rewritten in terms of occupied ( $\psi$ i $\psi \mathrm{i})$ and virtual ( $\psi \mathrm{a} \psi \mathrm{a})$ orbitals as

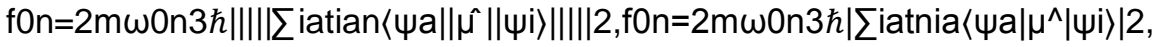

where the amplitudes tiantnia are the coefficients from the eigenvectors in Eq. (8).

Collective excitations which involve mixing of different orbital manifolds (e.g., core and valence) are generally assumed to factorize for the hole component in the hard $x$-ray regime. This leads to a reduction in the size of 
the matrix which must be diagonalized as excitations are restricted to originate only from selected core orbitals. In the EUV range, it is not immediately clear that this is a valid approximation, and we refrain from making any assumptions about the separability. Additionally, for any excitations relatively high above the core excitation threshold, the basis setlimitations remain a serious issue.

The primary advantage of the Casida formalism is that it yields explicit states. This makes further characterization of the excitations very straightforward and allows for the possibility of investigating the electronic forces on the nuclei and even excited state molecular dynamics. However the cost and difficulties of describing high energy states with a localized basis set make it less attractive for computing EUV spectra, particularly for condensed phase systems.

Another route to evaluate the LR-TDDFT equations, which avoids the difficulties associated with describing empty orbitals, is using the Lanczos algorithm. First, the TDKS equations (Eq. (3)) are rewritten in the form of the Liouville-von Neumann equation for the density $\rho(t) \rho(t)$,

$i \partial \rho(t) \partial t=[H K S(t), \rho(t)] . i \partial \rho(t) \partial t=\left[H^{\wedge} K S(t), \rho(t)\right]$

Then using the standard expressions for the linear response of the density and the Hamiltonian, the linearized version of Eq. (16) is obtained,

$i \partial \rho(1)(t) \partial t=[H(0) K S, \rho(1)(t)]+[\operatorname{Vext}(t), \rho(0)]+[\operatorname{VHXC}(t), \rho(0)], i \partial \rho(1)(t) \partial t=\left[H^{\wedge} K S(0), \rho(1)(t)\right]+[\operatorname{Vext}(t), \rho(0)]+[\operatorname{VHXC}(t), \rho(0)]$,

where $\rho(t)=\rho(0)+\rho(1)(t) \rho(t)=\rho(0)+\rho(1)(t), \rho(0) \rho(0)$ is formed from solutions to the ground state system $\hat{\mathrm{H}}(0) \mathrm{KSH}^{\wedge} \mathrm{KS}(0), V_{\text {ext }}$ is the perturbation potential, and $V H X C$ is the linear response of the Hartree plus XC potential induced by $\rho(1)(t) \rho(1)(t)$,

$\operatorname{VHXC}(\boldsymbol{r}, \mathrm{t})=\iint\left(1\left|r-r^{\prime}\right| \delta\left(t-t^{\prime}\right)+\delta v X C(r, t) \delta \rho(r, t)\right) \times \rho(1)\left(r^{\prime}, t^{\prime}\right) d r^{\prime} d t^{\prime} . V H X C(r, t)=\iint\left(1\left|r-r^{\prime}\right| \delta\left(t-t^{\prime}\right)+\delta v X C(r, t) \delta \rho(r, t)\right) \times \rho(1)\left(r^{\prime}, t^{\prime}\right) d r^{\prime} d t^{\prime}$

Defining the Liouvillian super-operator $\mathcal{L} L$ as

$\mathcal{L} \cdot \rho(1)=[\hat{H}(0) K S, \rho(1)]+[\operatorname{VHXC}[\rho(1)], \rho(0)] L \cdot \rho(1)=\left[H^{\wedge} K S(0), \rho(1)\right]+[\operatorname{VHXC}[\rho(1)], \rho(0)]$

and Fourier transforming Eq. (17) results in the expression

$(\omega-\mathcal{L}) \cdot \rho(1)(\omega)=[\operatorname{Vext}(\omega), \rho(0)] \cdot(\omega-L) \cdot \rho(1)(\omega)=[\operatorname{Vext}(\omega), \rho(0)]$

For an external potential of the form $\operatorname{Vext}(\boldsymbol{r}, \omega)=-E(\omega) \cdot \boldsymbol{r} \operatorname{Vext}(\mathbf{r}, \omega)=-E(\omega) \cdot \mathbf{r}$, the frequency dependent polarizability (gas phase) or susceptibility (solid state) can be written as

$\alpha \mathrm{ij}=-\operatorname{Tr}(\mathrm{ri}(\omega-\mathcal{L})-1 \cdot[\mathrm{rj}, \rho(0)]) \cdot \alpha \mathrm{ij}=-\operatorname{Tr}(\mathrm{ri}(\omega-\mathrm{L})-1 \cdot[\mathrm{rj}, \rho(0)])$.

Recalling that the absorption spectrum is directly related to the polarizability/susceptibility as shown in Eq. (12), defining $s j=[r j, \rho(0)] s j=[r j, \rho(0)]$ and $\langle A|| B\rangle=\operatorname{Tr}(A \dagger B)\langle A \mid B\rangle=\operatorname{Tr}(A \dagger B)$, and rewriting once more,

$\alpha \mathrm{ij}=-\langle\mathrm{ri}||(\omega-\mathcal{L})-1 \cdot \mathrm{sj}\rangle, \alpha \mathrm{ij}=-\langle\mathrm{ri} \mid(\omega-L)-1 \cdot \mathrm{sj}\rangle$,

highlights that the polarizability is defined by off-diagonal elements of the resolvent of the Liouvillian superoperator. An elegant method of solving an equation of the form 
$g(\omega)=\langle u|(\omega-A)-1) v\rangle g(\omega)=\langle u|(\omega-A)-1) v\rangle$

is through the Lanczos-Haydock recursion method, in which one recursively forms a tri-diagonal matrix to solve directly for $g(\omega) g(\omega)$. In this case, one obtains the entire spectrum, without solving for explicit states, and the computational cost is dictated by the number of iterations that must be performed to achieve convergence of the spectrum.

Instead of using a localized atomic basis as is usually done in the Casida formalism, for the Lanczos method a planewave basis is used, which can be systematically improved by increasing the planewave energy cutoff (complete in the limit of an infinite cutoff). Due to the high energies required to describe core electrons, pseudopotentials are generally used with planewave basis setswhere the inner-

shell electrons are replaced with a smooth potential. At EUV energies, it is necessary to exclude all accessible semi-core electrons from the pseudopotential, which results in the necessity of higher energy cutoffs. The computational cost associated with high energy cutoffs and the sensitivity of the intensity to the selected pseudopotential constitute the primary drawbacks when using the Lanczos algorithm for LR-TDDFT. However, especially for condensed phase systems, it is currently the most efficient method for obtaining EUV excitations and will be used for the primary results in this paper.

2. Real time propagation

An alternative approach to TDDFT is applying a propagator to the initial state of the system and allowing it to evolve in time. Generally, this means starting from the ground state DFT solutions and numerically applying a propagator to the wave-function,

$\left.\| \psi \mathrm{i}(\boldsymbol{r}, \mathrm{t})\rangle=\mathrm{U}^{\Upsilon}(\mathrm{t}, 0) \| \psi \mathrm{i}(\boldsymbol{r}, 0)\right\rangle .|\psi \mathrm{i}(\mathbf{r}, \mathrm{t})\rangle=\mathrm{U}^{\wedge}(\mathrm{t}, 0)|\psi \mathrm{i}(\mathbf{r}, 0)\rangle$.

The time-propagation operator $\hat{U} U^{\wedge}$ may be written as a time-ordered product, $\mathrm{U}(\mathrm{t}, 0)=\sum \mathrm{n}=0 \infty(-\mathrm{i}) \mathrm{nn} ! \mathrm{t} \mathrm{t} 0 \mathrm{~d} \tau 1 \int \mathrm{t} 0 \mathrm{~d} \tau 2 \ldots \times \int \mathrm{t} 0 \mathrm{~d} \tau \mathrm{n} \mathcal{T}\{\mathrm{H}(\tau 1) \mathrm{H}(\tau 2) \ldots \mathrm{H}(\tau \mathrm{n})\}, \mathrm{U}(\mathrm{t}, 0)=\sum \mathrm{n}=0 \infty(-\mathrm{i}) \mathrm{nn} ! \int 0 \mathrm{td} \tau 1 \int 0 \mathrm{td} \tau 2 \ldots \times \int 0 \operatorname{td} \tau \mathrm{n} T\left\{\mathrm{H}^{\wedge}(\tau 1) \mathrm{H}^{\wedge}(\tau 2) \ldots \mathrm{H}^{\wedge}(\tau n)\right\}$, which for a time-independent Hamiltonian reduces to

$U^{\wedge}(t, 0)=e-i H r . U^{\wedge}(t, 0)=e-i H^{\wedge} t$.

In TDDFT, the Hamiltonian is not time-independent due to the external field. Various approaches have been used for approximating the time evolution operator, and many of these are detailed in the paper by Castro, Marques, and Rubio.

The frequency dependent polarizability, and ultimately the optical absorption spectrum, can be obtained directly from the real-time propagation. As the wavefunction is propagated, the time-dependent response of the dipole is determined and Fourier transformed into the frequency domain,

$\alpha z(\omega)=e 2 \hbar k \int d \operatorname{dei} \omega t \sum \mathrm{i}\langle\phi i(t)\|\hat{z}\| \phi i(t)\rangle . \alpha z(\omega)=e 2 \hbar k \int d t e i \omega t \sum i\left\langle\varphi i(t)\left|z^{\wedge}\right| \varphi i(t)\right\rangle$.

In order to reproduce the linear response spectrum, only the first order response of the wavefunction is retained.

The primary benefits to RT-TDDFT are that it only requires occupied orbitals, and that it can be used for spectra beyond the linear-response regime. It does have a very large prefactor and can be very computationally intensive even for small systems. For EUV excitations, the necessary short time steps and consequently long simulation times needed to converge the spectra mean that it is still cost limited for the types of condensed phase materials we ultimately hope to study. Although much progress has been made in this area in recent years (e.g., Refs. 56-58), RT-TDDFT is not yet routinely usable for large systems in the EUV range, and as discussed in Sec. $\underline{\text { A 1 }}$, it is not desirable to restrict excitations to specific subspaces, which provides one mechanism for lowering the cost. Various types of basis sets can be used for RT-TDDFT, but for the condensed phase photoresistapplications that are the ultimate goal of this paper, localized basis sets will be quite inefficient. The implementation of RT-TDDFT that we use is in the Octopus code, which computes the wavefunction using a uniform real-space grid and allows us to use the same pseudopotentials as those used for the Lanczos algorithm approach. This grid must be quite fine for localized EUVexcitations, and 
in order to converge the spectrum, the time step should not be larger than $1 / \omega m a x 1 / \omega m a x$, where wmaxwmax corresponds to the highest frequency in the desired spectrum.

\section{B. Computational methods}

We explore the use of TDDFT for computing absorption spectra in the EUV region. Two different linearresponse methods are used: direct Davidson diagonalization of the TDDFT equations using a localized basis set and the Lanczos approach with a planewave basis. Formally either type of basis set can be used with both methods; however, it is impractical to use direct diagonalization with the extremely large planewave basis sets. Furthermore, as currently formulated, the Lanczos method requires the use of a planewave basis set, which also provides a more efficient representation of orbitals and responses at energies high above the ionization threshold.

All localized basis set calculations were performed using QCHEM, and plane-wave calculations used QUANTUMESPRESSO, and its TURBOTDDFT module.

For the localized basis calculations, various basis sets and functionals were considered. The basis sets are generated for this work based off the def2-QZVPD basis, which uses a small-core effective potential for heavy elements such as iodine. The effective core potential (ecp) allows the incorporation of relativistic effects and reduces the cost by not explicitly treating the deepest inner-shell electrons. Basis sets with additional functions were generated and details are included as the supplementary material. All geometry optimizations were carried out using B3LYP/cc-pVDZ (using the corresponding effective core potentials for $\mathrm{Br}$ and I). All other calculations use the PBE functional unless otherwise noted. The effect of using other functionals, including hybrids and range-separated hybrids, was explored, but the changes they induce in broad features that occur well above any absorption edges appear to be negligible and do not justify the additional computational effort. Additional details on the functional dependence of the excitation are included in the supplementary material. For the plane-wave calculations, the energy cutoff was set at $100 \mathrm{Ry}$, and the unit cell was $20 \AA \times 20 \AA \times 20 \AA$, unless indicated otherwise. The PBE functional was used for all calculations, and optimized norm-conserving Vanderbilt (ONCV) pseudopotentials were used. For Br and I, pseudopotentials incorporating the $d$-electrons are required. The norm-conserving pseudo-potentials (NCPPs) used are included with the Octopus code distribution. The outermost shell and any adjacent delectrons are included as valence electrons, and two projectors are used for each value of the angular momentum. A core correction is not included. In all absorption spectra, the broadening is set to a constant value of $1 \mathrm{eV}$, and a minimum of 2000 Lanczos iterations (extrapolated to 50000 iterations) were computed. It is necessary to use norm-conserving pseudopotentials (NCPPs) to reliably capture the absorption intensity in the EUV range. Initially ultra-soft pseudopotentials (USPPS) were used, but the ones that were used severely underestimated the intensity due to excitation of the $d$-electrons. Figure $\underline{4}$ illustrates the loss of amplitude associated with the $d$-electron wavefunctions that can occur when using USPPs. Although it is possible to reduce the cutoff radius in generating the USPPS to reduce the loss of intensity, this increases the required plane-wave cutoff and eliminates the primary benefit of using USPPS.

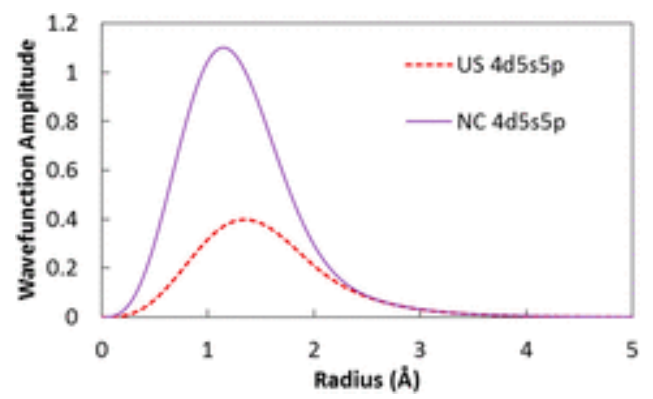

FIG. 4.lodine $4 d$ wavefunctions from ultrasoft and norm conserving pseudopotentials. The ultrasoft pseudopotential has very low intensity near the nucleus and results in a loss of oscillator strength.

RT-TDDFT results were computed using the OcTOPUs code. A grid of $0.12 \AA$ was used and the wavefunctions were propagated with a time step of 0.001 atomic units $(2.4 \times 10-52.4 \times 10-5 \mathrm{fs})$ using the approximately enforced time reversal symmetry (aetrs) propagator. Other propagators were also explored and gave very similar results. 10000 time steps were computed and used to form the spectra. 


\section{Validation: Methane, methanol, and the methyl halides}

Before proceeding to discuss the halogenated phenol resist analogues, which are the primary focus of this paper, we begin with a series of substituted methanes $(\mathrm{CH} 3 \mathrm{XCH} 3 \mathrm{X}: \mathrm{X}=\mathrm{H}, \mathrm{OH}, \mathrm{F}, \mathrm{Cl}, \mathrm{Br}, \mathrm{I})$ in the gas phase for which experimental spectra exist. This will allow assessment of the successes and limitations for the theoretical models of computing the absorption spectra.

The linear response Lanczos spectra for gas phase methyls computed with TURBOTDDFT in QUANTUMESPRESSO are shown in Fig. $\underline{5}$. The primary peak occurs near $20 \mathrm{eV}$ for all species and is due to excitations of the valence electrons. The onset energy corresponds to the gap between the HOMO and the LUMO. The valence peaks for methanol and methyl fluoride are significantly broader than the others, which is due to excitations of deeper valence electrons. The computed orbital energies for each of these molecules are represented in Fig. $\underline{6}$.

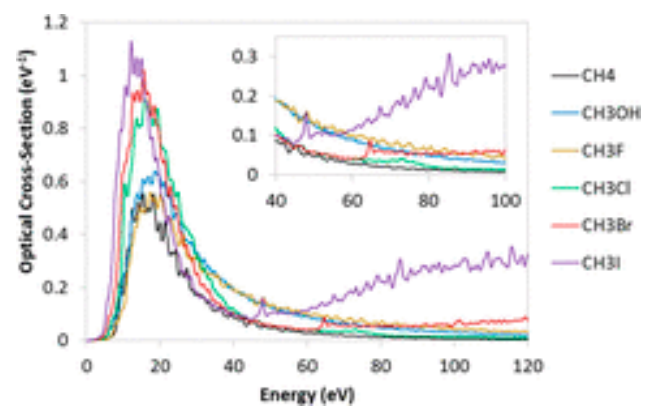

FIG. 5.Computed optical absorption for $\mathrm{CH} 4 \mathrm{CH} 4, \mathrm{CH} 3 \mathrm{OHCH} 3 \mathrm{OH}, \mathrm{CH} 3 \mathrm{FCH} 3 \mathrm{~F}, \mathrm{CH} 3 \mathrm{ClCH} 3 \mathrm{Cl}, \mathrm{CH} 3 \mathrm{BrCH} 3 \mathrm{Br}$, and $\mathrm{CH} 3 \mathrm{ICH} 3 \mathrm{I}$ from 0 to $120 \mathrm{eV}$. Note the significantly increased intensity for methyl iodide at $92 \mathrm{eV}$.

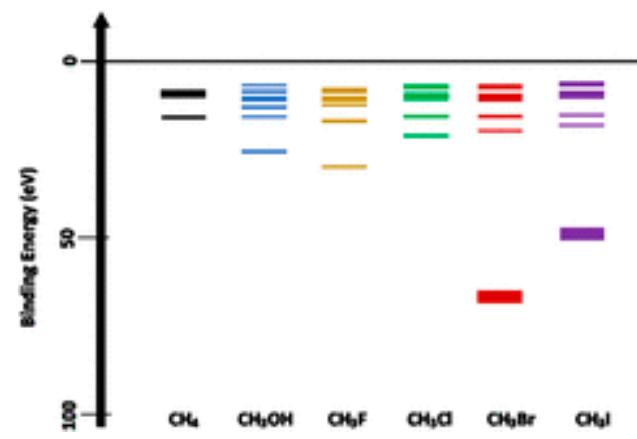

FIG. 6.Valence and semi-core orbital energies for methyl compounds up to $92 \mathrm{eV}$ binding energy computed with PBE/Def2-

QZVPD. $\mathrm{CH} 3 \mathrm{OHCH} 3 \mathrm{OH}$ and $\mathrm{CH} 3 \mathrm{FCH} 3 \mathrm{~F}$ have slightly deeper valence levels than the other compounds, while $\mathrm{CH} 3 \mathrm{BrCH} 3 \mathrm{Br}$ and $\mathrm{CH} 3 \mathrm{ICH} 3 \mathrm{I}$ have semi-core $3 d$ and $4 d$ levels, respectively.

In addition to the valence molecular orbitals formed from $s$ and $p$ atomic orbitals, methyl iodide and methyl bromide both possess semi-core atomic-like $d$-orbitals. These $d$ orbitals have binding energies much larger than the valence electrons, but smaller than the inner-core electrons, and can be ionized with EUV radiation. For elemental bromine, the $3 d$ electrons are bound by $69\left(3 d_{5 / 2}\right)$ and $70\left(3 d_{3 / 2}\right) \mathrm{eV}$, and for iodine, the $4 d$ electrons are bound by $48.9\left(4 d_{5 / 2}\right)$ and $50.6\left(4 d_{3 / 2}\right) \mathrm{eV}$. In the absorption spectrum (Fig. $\left.\underline{5}\right)$, small peaks near $70 \mathrm{eV}$ and $50 \mathrm{eV}$ are present as expected for $\mathrm{CH} 3 \mathrm{BrCH} 3 \mathrm{Brand} \mathrm{CH} 3 \mathrm{ICH} 3 \mathrm{I}$, respectively. It should be noted that our calculations do not currently include spin-orbit coupling, so we do not reproduce the splitting for the $d$-orbitals. In iodine, most of the oscillator strength associated with the $4 d$-electrons actually occurs at energies significantly above the onset threshold. This is a well-known phenomenon related to the poor overlap with the final state $f$-orbitals at the ionization threshold. ${ }^{69,70}$ For iodine, the peak of this intensity associated with excitation from the $4 d$-orbitals occurs around $90-100 \mathrm{eV}$. It is also worth noting that the discrete peaks near the $4 d$ ionization threshold are due to $4 \mathrm{~d} \rightarrow 6 \mathrm{p} 4 \mathrm{~d} \rightarrow 6 \mathrm{p}$ transitions, and the broad intense peak arises from 
the $4 \mathrm{~d} \rightarrow \mathrm{nf} 4 \mathrm{~d} \rightarrow \mathrm{nf}$ transitions. The methyl bromide spectrum also displays a delayed onset in the peak photoabsorption intensity, but it does not peak until nearly $200 \mathrm{eV}$, which is outside the EUV range of interest. At the critical EUV photon energy, $92 \mathrm{eV}$, methyl iodide absorbs photons most efficiently, followed by methyl fluoride and methanol. The other species containing heteroatoms are nearly identical, and methane has the lowest absorption. For methyl fluoride and methanol, which have more strongly bound $2 \mathrm{~s}$ states, the increased intensity at $92 \mathrm{eV}$ is due solely to the slow decay of the valence peak; however, for methyl iodide, the increased intensity is due to the opening of the 4dionization channels.

To assess the performance of the Lanczos approach, we compare directly to the absolute cross sections experimentally determined by Brion et al. (see Fig. 7) ${ }^{13,63-67}$ Also included are the RT-TDDFT results. Generally the experimental and theoretical spectra match up quite well. The theoretical spectra slightly overestimate the valence contribution and do not decay as rapidly after $\approx 100 \approx 100 \mathrm{eV}$. However in the neighborhood of $92 \mathrm{eV}$, the absolute cross section is quite reasonable compared to experiment. The RTTDDFT method produces spectra that are nearly identical to those generated using the Lanczos algorithm. The real-time results are significantly more computationally intensive, and although RT-TDDFT should ultimately scale linearly with system size, it has a very large computational prefactor and this linear scaling is not realized in the systems we are investigating. Additionally, in order to converge the spectrum in the EUV range, a very short time step and fine grid are required, which also drives up the computational cost.

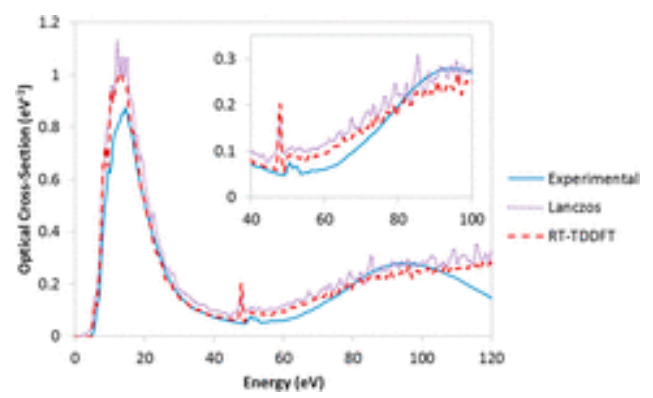

FIG. 7.Comparative optical absorption for $\mathrm{CH} 3 \mathrm{ICH} 3 \mathrm{I}$ computed using the Lanczos algorithm (QuantumESPRESSO), real-time TDDFT (Octopus), and experimental spectrum from the work of Brion et al. $\stackrel{13}{ }$ See text for further details.

We also computed the spectrum using Casida's sum-over-states formalism primarily to obtain information about individual transitions. Figure $\underline{8}$ shows the breakdown of the spectral intensity for $\mathrm{CH}_{3}$ for valence and semi-core excitations, as well as excitations which are defined as mixed (amplitude components of TDDFT states describing excitations from semi-core orbitals greater than $33 \%$ and less than $67 \%$ ). Initially, working within the Tamm-Dancoff approximation, Fig. $\underline{8(a)}$, it was seen that there was significant mixing between the semi-core and valence states; however, when full LR-TDDFT is used, this mixing appears to be essentially eliminated, Fig. $\mathbf{8 ( b )}$. This mixing is also replicated in the iodide anion and is an atomic effect. Thus, the separation of excitations from the semi-core and valence orbitals when using full TDDFT and the excitations are restricted to originate either from the $\mathrm{I}(4 d)$ orbitals or the molecular valence subspaces and are treated independently. The computed spectrum using this restricted window DFT is shown in Figure $\underline{9}$. Full TDDFT is used for all results in this paper with the exception of the spectrum as shown in Fig. $\underline{\text { 8(a). }}$.

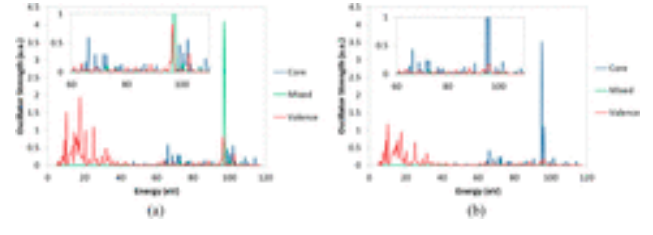

FIG. 8.lodomethane spectrum broken into individual states for (a) TDA/PBE/def2-QZVPD and (b) TDDFT/PBE/def2-QZVPD. Core states refer to ionization from I $(4 d)$ atomic orbitals, and valence states arise from are all other orbitals with smaller binding energies. The mixed states have both core and valence characters (defined as above $33 \%$ and below $67 \%$ core character). The TDA results in mixing between the core and valence states which is removed with full LR-TDDFT. 


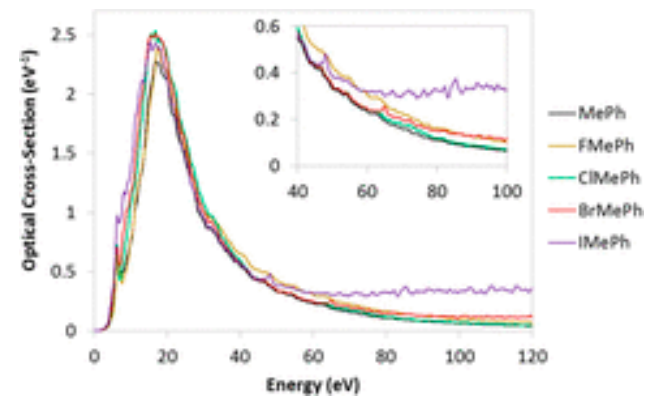

FIG. 9.Absorption for $\mathrm{CH} 3 \mathrm{ICH} 3 \mathrm{I}$ computed using LR-TDDFT within the Casida formalism as implemented in Q-chem. Experimental spectrum also included for comparison. The computed spectrum is comprised of excitations from the I(4d) semi-core orbitals (red) and excitations from the valence band (blue). The peaks are broadened by $1 \mathrm{eV}$ for the separate spectra and 7 eV for the combined spectrum (see text for additional details).

The primary drawback to using localized basis sets is that they do not describe the continuum functions properly and it is not practical to fully converge the spectrum with respect to the basis set due to the rapid increase in memory requirements. Particularly because increasing the basis set also increases the number of states that must be computed. The lower energy range of the spectrum converges rapidly, but higher excitations are still not fully converged even with the largest basis set considered: $\mathrm{H}(6 s 6 p 4 d 1 f), \mathrm{C}(10 s 6 p 6 d 2 f)$, and I(10s9p7d4f). For the spectrum of methyl iodide, without separating the electronic spaces, it is not possible to reach $92 \mathrm{eV}$ on a node with $64 \mathrm{~GB}$ of memory with this basis, however separating them allows fewer roots to be computed in each calculation.

Although excitations into the vacuum continuum are completely neglected when using a localized basis, the Casida method has the advantage that individual states are readily available, which is useful for obtaining a better understanding of the nature of the individual states. Incorporating finite lifetime effects through absorptive boundary conditions or other methods will certainly improve the shape of the spectrum, but ultimately we find that it is much more efficient to use a plane-wave basis set for the spectrum and primarily focus on the Casida method as a means of rapidly determining the contributions of single particle excitations to the excitation spectrum.

All electrons with binding energies less than $92 \mathrm{eV}$ can potentially be ionized; however, the dominant contribution is due to semi-core and deeper valence electrons in $\mathrm{CH} 3 \mathrm{ICH} 3$. This is a consequence of the resonant enhancement term in Eq. (1). For $\mathrm{CH} 3 \mathrm{FCH} 3 \mathrm{~F}$ and $\mathrm{CH} 3 \mathrm{OHCH} 3 \mathrm{OH}$, which have very broad valence peaks, it is also true that the dominant contribution is due to the deeper valence levels at EUV energies. Photoionization preferentially removes electrons in the deep valence and semi-core levels, unlike electroninduced ionization that primarily affects the outer valence electrons. This is partly because the inner electrons are shielded from incoming charged particles, but uncharged radiation is able to penetrate deeper into the core. Thus, EUV photon ionization is likely to create holes that are deeper and more localized than ionization by high energy electrons.

\section{RESULTS AND DISCUSSION}

In this section, we will only be using the Lanczos method to compute the absorption of 4-X-2-methyl phenols (X $=\mathrm{H}, \mathrm{F}, \mathrm{Cl}, \mathrm{Br}$, l; also denoted XMePh) as shown in Fig. 2(b). Even for such relatively small molecules, solving for the required number of eigenstates to obtain excitations near $92 \mathrm{eV}$ with a sufficiently sized basis set is computationally demanding, likewise RT-TDDFT is quite expensive and should produce results that are similar to the Lanczos method.

\section{A. Gas phase results}

The gas phase absorption spectra for each of the phenols are shown in Fig. 10. In many respects the variation due to the addition of a halogen appears very similar to that obtained with the methyl series. Overall, the primary difference is due to the halogen contributing to a smaller percentage of overall absorption in the molecule. The total intensity in optical absorption is determined by the number of electrons in the system (Thomas-Reiche-Kuhn Sum rule); thus, while the peak intensity of methane (8 valence $\mathrm{e}^{-}$) is roughly half that 
of methyl chloride (14 valence $\mathrm{e}^{-}$), the difference between MePh and CIMePh in the valence band is nearly imperceptible.

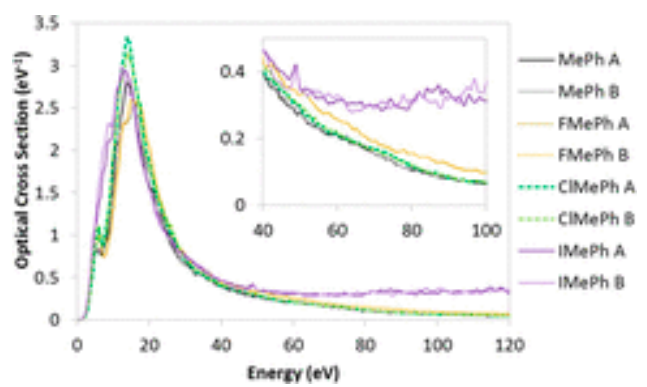

FIG. 10.Computed optical absorption spectra for the methyl phenols, XMePh $(X=\mathrm{H}, \mathrm{F}, \mathrm{Cl}, \mathrm{Br}, \mathrm{I})$ from 0 to 120 eV.

The onset of the valence electron peak is nearly the same for all the phenols discussed in this paper, indicating that the HOMO-LUMO gap is nearly the same for all of them. However, there is a significant variation from 8 to $25 \mathrm{eV}$, with the heavier halogens causing a red shift in the absorption.

At $92 \mathrm{eV}$, the absolute intensity for both methyl, Fig. $\underline{5}$, and phenol, Fig. $\underline{10}$, species is nearly identical. This is consistent with a very small oscillator strength contribution from valence electrons to excitations in the high energy range. EUV absorption for both $\mathrm{CH} 3 \mathrm{ICH} 3 \mathrm{I}$ and IMePh appears almost entirely dominated by excitations from the single iodine atom.

\section{B. Condensed phase model}

As resist materials are condensed phase systems, we investigated a model for a molecular crystal of the phenols. We are not yet attempting to model any of the complexity present in a true photoresist, but rather to investigate the effect on absorption of moving from the gas phase to a condensed phase system. A supercell consisting of three monomer units was formed for each species, and the geometry and unit cell parameters were relaxed. A total of 4-5 local ground state minima were computed for each $\mathrm{XMePh}(\mathrm{X}=\mathrm{H}, \mathrm{F}, \mathrm{Cl}, \mathrm{I})$, as well as the corresponding absorption spectra. As there was no significant variation in the absorption at $92 \mathrm{eV}$ between the different geometries, only two local minima for each material are shown in Fig. 11 and the different optimized geometries are denoted A and B.

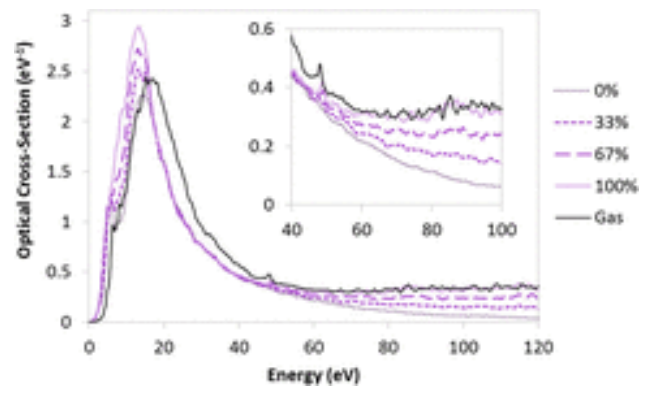

FIG. 11.Computed optical absorption for the condensed phase halogenated methylphenols from 0 to $120 \mathrm{eV}$, these are all normalized to the number of monomers and the size of the unit cell. Two different local minima A and B are shown for each species.

There is some variation in the valence peak between the different geometries, which is particularly obvious in the case of IMePh. As the valence electrons are quite delocalized, they are expected to be the most affected by changes in geometry as the orbitals become distorted. At $92 \mathrm{eV}$, the effect on the spectrum due to different geometries is quite small, which also supports the conclusion that these excitations are mostly atomic-like. For IMePh, the condensed and gas phase spectra are shown in Fig. 12. Here the effect of moving to the condensed phase in the valence region is especially pronounced. The valence peak for the condensed model is significantly narrower and red shifted with respect to that of the gas phase. At the onset of the I(4d) peak, there is also a small shift in the main peak between the gas and condensed phase materials. The gas phase results appear to be fully converged with respect to the unit cell size; however, it is possible that there are periodic boundary effects in the condensed phase cell that result in the $\mathrm{d}$ electrons being more strongly bound. 
Beyond about $60 \mathrm{eV}$, the gas and condensed phase cross sections are essentially equivalent within numerical accuracy. This supports the conclusion that the atomic composition of the material and not the specific molecular arrangement is the dominant effect for EUV absorption. However, it should be recalled that the geometry may still have a large effect on the processes which occur in photoresists subsequent to EUV excitation.

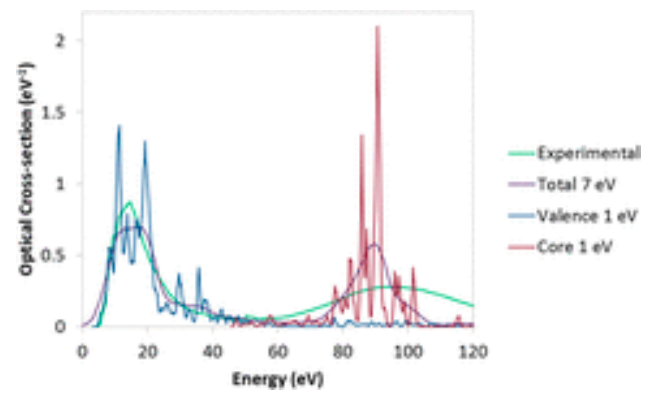

FIG. 12. Computed optical absorption for the condensed phase iodo-methylphenol from 0 to 120 eV with decreasing numbers of iodine atoms (IMePh-A) and for gas phase IMePh. This is shown as intensity/molecule and normalized by unit cell size.

The effect of removing iodine atoms individually was also investigated. The results for the "A" geometry are shown in Fig. 12. The spectra are normalized to the number of molecules, and thus the total absorption of the fully iodated supercell matches very closely with the isolated gas phase molecule for EUV excitation.

Replacing a halogen with a hydrogen on one of the IMePh species in the unit cell results in a material where $67 \%$ of the molecules are IMePh and the others are simply MePh. Replacing two or three halogens results in a $33 \%$ or $0 \%$ iodated material, respectively. In this case, adding a single iodine atom (33\% iodation) fortuitously has roughly the same contribution as the decaying intensity of the valence band. More importantly, if the valence contribution is subtracted out, the intensity increases linearly with iodation (at $92 \mathrm{eV}, R^{2}=0.998$ ). This is again consistent with the absorption of EUV radiation being dominated by atomic-like contributions.

\section{Relation to atomic data}

Based on the preceding discussion, it indeed seems reasonable to conclude that the absorption spectra at EUV energies are dominated by atomic contributions, and not strongly influenced by the specific structure of the material. Although, we note that this is only justified when discussing materials where the oxidation state does not change based on the bonding of the atoms. There is likely to be a significant change in the EUV absorption for atoms which have different oxidation states at different geometries, which should be revisited in the context of inorganic resist materials. This expected sensitivity to oxidation state is analogous to the behavior that is observed for higher energy x-ray absorption as in XANES.

As it is a common practice to simply use semi-empirical atomic optical cross sections in the resist community,

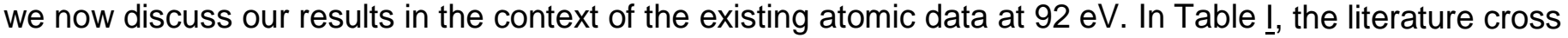
sections (Henke values) are converted to values for isolated atoms, and then the total cross section is computed as a sum of the atomic cross sections. The experimental values are also given for the methyl series.

TABLE I. Comparison of total optical cross sections in $\mathrm{eV}^{-1}$ determined from literature values with computed values in this work at $92 \mathrm{eV}$.

TABLE I.Comparison of total optical cross sections in $\mathrm{eV}^{-1}$ determined from literature values with computed values in this work at $92 \mathrm{eV}$.

\begin{tabular}{l|l|l|l} 
& Henke & This work & Experiment \\
\hline $\mathrm{CH} 4 \mathrm{CH} 4$ & 0.0075 & 0.0091 & 0.0056 \\
\hline
\end{tabular}




\begin{tabular}{|c|c|c|c|}
\hline & Henke & This work & Experiment \\
\hline $\mathrm{CH} 3 \mathrm{OHCH} 3 \mathrm{OH}$ & 0.031 & 0.036 & 0.024 \\
\hline $\mathrm{CH} 3 \mathrm{FCH} 3 \mathrm{~F}$ & 0.043 & 0.050 & 0.033 \\
\hline $\mathrm{CH} 3 \mathrm{ClCH} 3 \mathrm{Cl}$ & 0.020 & 0.015 & 0.013 \\
\hline $\mathrm{CH} 3 \mathrm{BrCH} 3 \mathrm{Br}$ & 0.033 & 0.060 & 0.022 \\
\hline $\mathrm{CH} 3 \mathrm{ICH} 3 \mathrm{I}$ & 0.26 & 0.28 & 0.27 \\
\hline MePh & 0.070 & 0.080 & $\ldots$ \\
\hline FMePh & 0.11 & 0.12 & $\ldots$ \\
\hline ClMePh & 0.082 & 0.085 & $\ldots$ \\
\hline BrMePh & 0.095 & 0.13 & $\ldots$ \\
\hline $\mathrm{IMePh}$ & 0.32 & 0.35 & $\ldots$ \\
\hline
\end{tabular}

From the methyl series, where the experimental values exist, it can be seen that the literature values overestimate the atomic contributions, with the exception of $\mathrm{CH} 3 \mathrm{ICH} 3 \mathrm{I}$. In our calculations, we also overestimate the experimental values, however in some cases our values are slightly closer to the experimental results than those obtained with Henke's data. We do quite well for $\mathrm{CH} 3 \mathrm{ICH} 3 \mathrm{I}$ which is dominated by the semi-core contribution, whereas in the other cases, the total cross sections are highly dependent on the intensity from the decaying tail of the valence excitations. A possible explanation for the overestimated valence tail is that significant excitionic effects in the optical absorption spectrum would shift more of the oscillator strength to lower energies, resulting in a reduced intensity at higher energies (total area must be conserved by the Thomas-Reiche-Kuhn sum rule). Additionally, TDDFT generally spreads oscillator strength over a wider energy range than higher order approaches (e.g., Bethe-Salpeter). 
It appears that using atomic data is generally sufficient for estimating EUV absorption. However, it may be worth revisiting the generation of the 1993 atomic data, as there may now be more accurate methods available than the semi-empirical approach employed more than two decades ago. Additionally it should be noted that while the atomic data appear valid for EUV and higher energies, the approximation breaks down rapidly at lower energies when the valence electrons provide the primary contributions to the excited states.

\section{CONCLUSIONS}

Accurately computing optical absorption spectra at EUV energies is an extremely challenging task due to the large number of excited states that must be computed. Additionally, our task is ultimately to develop a general method which can be applied to condensed phase photoresist materials, which currently have an indeterminate composition.

Restricted window methods that limit the allowed excitations do exist and are used extensively for $x$ ray absorption calculations with localized basis sets, but for EUV absorption it was not desirable to limit the allowed excitations before running the calculations. This is primarily due to the fact that the energetic separation between the valence and core orbitals is not sufficiently large to automatically justify the separation of valence and semi-core orbitals, and significant mixing does occur, as seen in Fig. $\underline{8}$, within the TDA approximation, although the mixed states disappear when full TDDFT is used. Furthermore, for atoms like fluorine, which have a significant EUVabsorption cross section but do not have semi-core electrons, such a separation is not possible. Generally localized basis set methods suffer from an inability to properly describe the continuum states and require impractically large basis sets to describe excitations above the onset threshold or without some other treatment such as absorbing boundary conditions or imaginary basis functions. Thus, we use the localized basis methods primarily to assess the character of the excitations.

As an alternative to computing the excitations as eigenvalues and eigenvectors of a matrix, we employed the Lanczos algorithm to compute the full spectrum by Fourier transforming the linear response of the system. This method does not directly allow for the investigation of individual states, or a simple way to determine the character of the bands. Although it is possible to compute the response of the density for a given excitation energy, this is not feasible over a wide range. This method is shown to produce essentially the same spectra as are obtained using RT-TDDFT at a much lower computational cost.

It generally seems reasonable to interpret EUV absorption as being atomic-like, which can be seen by comparing the absorption from the phenol and methyl species. Additionally, the difference between the gas and condensed phase models is negligible in the EUV range, further confirming the atomic-like nature. There may be benefits to revising the currently used atomic data with more accurate numbers, and then these values could be directly used to rapidly estimate the total absorption of a photoresist material.

For those designing EUV resists, we conclude that adding elements with high cross sections in the EUV range increases the absorption. Although closely related to the semi-core binding energies, the binding energies alone cannot be used directly to determine high EUV cross sections due to the possibility of delayed maxima. This is illustrated by the comparison of bromine, which has delectrons at $70 \mathrm{eV}$, and iodine, which has $d$ electrons at $50 \mathrm{eV}$. Based on Eq. (1), it may be expected that Br should have a higher EUV cross section than I, but the maximum absorption actually occurs at significantly higher energies (near $100 \mathrm{eV}$ for I and 200 $\mathrm{eV}$ for $\mathrm{Br}$ ). Additionally, considering elements with deep valence levels like fluorine also is seen to increase the EUV cross section, although this phenomenon is not due to the accessibility of additional channels, but simply due to the slower decay of the valence band cross section. Semi-core orbitals are atomically localized, so EUV ionization is very likely to create localized excited states, which may be useful for subsequent steps in the next generation of photolithography. The inner electronic structure is the most important aspect to consider in terms of improving EUV absorption, but ultimately the bonding and reactivity of the resist will also be critical in terms of the ability to pattern. 
SUPPLEMENTARY MATERIAL

See supplementary material for basis set coefficients used for the spectrum in Fig. $\underline{9}$ and additional details regarding the selection of the density functional.

\section{ACKNOWLEDGMENTS}

This work was performed under a user proposal at the Molecular Foundry at Lawrence Berkeley National Laboratory (LBNL), which is supported by the Office of Science, Office of Basic Energy Sciences, of the U.S. Department of Energy under Contract No. DE-AC02-05CH11231. Calculations were performed on the Molecular Foundry computing resources, Nano and Vulcan, managed by the High Performance Computing Services Group of LBNL. K.D.C. was supported by the Laboratory Directed Research and Development (LDRD) Program at LBNL, also supported under the same DOE contract. 